\title{
Editorial on the special issue of RTNS 2020
}

\author{
Sebastian Altmeyer ${ }^{1}$. Jean-Luc Scharbarg ${ }^{2}$
}

Published online: 20 February 2022

(c) The Author(s) 2022

The 28th edition of the Real-Time Networks and Systems Conference (RTNS) was without any doubt very different to previous editions of the conference. The 2020 edition had been planned as an intermediate step in the transition of the conference date from Autumn to Spring. The foreseen venue had been Paris, France. But the travel restriction and the corona pandemic restrictions have put an end to this plan. Instead, RTNS was organized as a purely virtual event. Nevertheless, RTNS 2020 presented 16 papers distributed over four sessions, a session on Real-Time Multicore Systems, on Timing and Monitoring, on Scheduling Systems and on Networked Systems, and a keynote given by Iain Bate from the University of York on "Timing Analysis and Verification of Multi-core Real-Time Systems for Aerospace Applications".

Papers recognized as outstanding papers from RTNS 2020 were invited to submit extended journal versions for this special issue of the Real-Time Systems Journal. In total, we invited three submissions that are presented in this issue after rigorous peer review by experts from the areas of real-time and networked systems. Two of the submissions investigate multiprocessor/multicore scheduling, although from fundamentally different perspectives and assumptions, the other paper provides a novel cache analysis which is fundamental to the estimation of worst-case execution time bounds.

The first paper in this special issue is "Workload assignment for global real-time scheduling on unrelated multicore platforms" by Antoine Bertout, Joel Goossens, Emmanuel Grolleau and Xavier Poczekajlo. The paper targets task assignment on modern MPSoCs based on a new system model and provides empirical evidence for its practical relevance.

The second paper, "Precise and Efficient Analysis of Context-Sensitive Cache Conflict Sets" by Florian Brandner, provides a novel cache and persistence analysis

Sebastian Altmeyer

altmeyer@informatik.uni-augsburg.de

Jean-Luc Scharbarg

jean-luc.scharbarg@toulouse-inp.fr

1 University of Augsburg, Augsburg, Germany

2 University of Toulouse, Toulouse, France 
for classical LRU caches as well as for method caches. The analysis is based on cache summaries, a novel approach to estimating the impact of portions of program code on the cache states.

The third paper, "Scheduling DAGs When Processor Assignments Are Specified" by Sanjoy Baruah, addresses the restricted assignment scheduling problem (RASP). First, the author proves that the scheduling problem is NP-hard in the strong sense, and then proceeds to identify conditions under which the problem can nevertheless be solved efficiently using integer linear programs.

We would like to thank all the authors that submitted their work to the special issue and express appreciation to the referees for their time and effort in selecting and improving the quality of the papers.

Funding Open Access funding enabled and organized by Projekt DEAL.

Open Access This article is licensed under a Creative Commons Attribution 4.0 International License, which permits use, sharing, adaptation, distribution and reproduction in any medium or format, as long as you give appropriate credit to the original author(s) and the source, provide a link to the Creative Commons licence, and indicate if changes were made. The images or other third party material in this article are included in the article's Creative Commons licence, unless indicated otherwise in a credit line to the material. If material is not included in the article's Creative Commons licence and your intended use is not permitted by statutory regulation or exceeds the permitted use, you will need to obtain permission directly from the copyright holder. To view a copy of this licence, visit http://creativecommons.org/licen ses/by/4.0/.

Publisher's Note Springer Nature remains neutral with regard to jurisdictional claims in published maps and institutional affiliations.

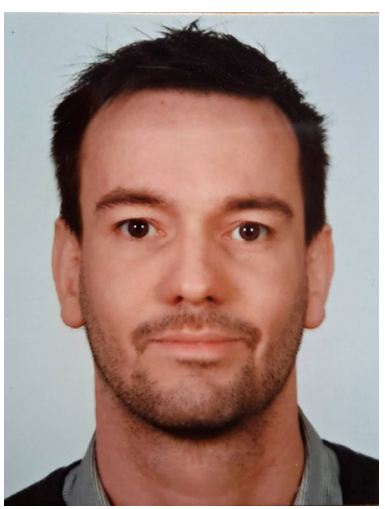

Sebastian Altmeyer is Full Professor at the University of Augsburg, Germany and head of the Chair for Embedded Systems. He received his $\mathrm{PhD}$ from Saarland University, Germany in 2012. Before he joined the University of Augsburg, he has been employed at the University of Amsterdam, The Netherlands, and the University of Luxembourg. Sebastian's research aims at the design and development of safe, reliable and performant embedded systems. This includes in particular real-time scheduling, worst-case execution time analysis and processor architectures for real-time systems. Visit es-augsburg.de/altmeyer for contact information and publications. 


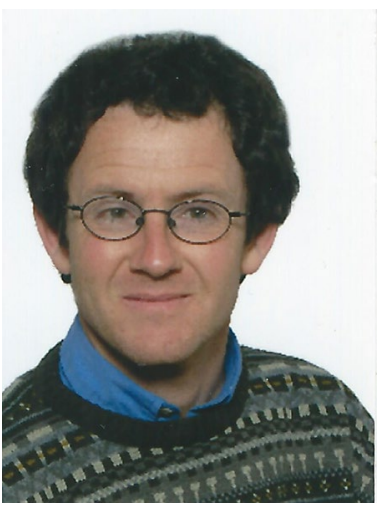

Jean-Luc Scharbarg received the $\mathrm{PhD}$ in computer science from the University of Rennes, France, in 1990 and the HDR from INP Toulouse in 2010. He has been associate professor at the University of Toulouse (INPT/ENSEEIHT and IRIT laboratory) between 2002 and 2012 and full professor since 2012. He has been head of the research team on network engineering of IRIT lab between 2011 and 2020. His current research interest concerns the analysis and performance evaluation of embedded networks, mainly in the context of avionics and automotive. He has co-authored more than one hundred papers in leading journals and conferences on real-time systems and networks. He has been involved in the organization of conferences and workshops on real-time networking. 